\title{
Relação peso-comprimento da corvina Micropogonias furnieri (Desmarest) (Pisces, Sciaenidae) na Baía de Sepetiba, Rio de Janeiro
}

\author{
Francisco Gerson Araújo ${ }^{1,2}$ \\ Rafaela Nascimento Vicentini ${ }^{1,3}$
}

\begin{abstract}
Length-weight relationship of white croaker Micropogonias furnieri (Desmarest) in the Sepetiba Bay, Rio de Janeiro. The length-weight relationship of populations of white croaker Micropogonias furnieri (Desmarest, 1823) in the Sepetiba Bay $\left(22^{\circ} 54^{\prime}-23^{\circ} 04^{\prime} \mathrm{S} ; 43^{\circ} 34^{\prime}-44^{\circ} 10^{\prime} \mathrm{W}\right)$, were assessed. It aims to contribute to the knowledge of the biometrics and to estimate the biomass of this very abundant fishery resource in the Bay. Fishes sampled, in both trawling beach seine, sampling programme, from October 1998 to May 1999 were analyzed. The equation found was $\mathrm{W}=0.00000942 \mathrm{~L}^{2.99}$ from the natural logaritmized transformation of the raw data, through ordinary least square equation $=\ln W=-11.57+2.996 \ln \mathrm{L}(\mathrm{r}=0.99)$. The condition factor was relatively high compared with coastal population from Southeast Brazil, probably due to in this study both males and females from juvenile and adult were considered, while in coastal areas, only adult female were analyzed. The alometry coefficient $(\theta)$, on the other hand, is very similar to the coastal Southeast Brazil population, but differed from Northeast and South Brazil populations.

KEY WORDS. Sciaenidae, length-weight relationship, coastal fishes, bays, Sepetiba Bay
\end{abstract}

Sciaenidae é representada por peixes costeiros, comumente encontrados em águas rasas da plataforma continental, próximas às desembocaduras de grandes rios, em fundos de areia ou lama. A espécie em estudo Micropogonias furnieri (Desmarest, 1823) (Sciaenidae), conhecida popularmente como corvina, constitui uma importante parcela das capturas comerciais efetuadas no sudeste do Brasil (MENEZES \& FIGUEIREDO 1980).

A relação peso-comprimento é um importante parâmetro em biologia de peixes, as suas aplicações incluem uma estimativa de uma destas variáveis, uma vez conhecida a outra (BEYER 1987). A conversão das equações de comprimento em peso para equivalentes de crescimento em peso, comparações morfométricas interespecíficas e interpopulacionais das espécies de peixes, e avaliação do índice de bem-estar das populações de peixes (BOLGER \& CONNOLLY 1989) são realizadas a partir da determinação da relação peso-comprimento. Essa relação também tem sido utilizada sob dois diferentes aspectos: estimar biomassas a partir de dados de

1) Laboratório de Ecologia de Peixes, Posto de Aqüicultura, Universidade Federal Rural do Rio de Janeiro. Antiga Rodovia Rio-São Paulo Km 47, 23851-970 Seropédica, Rio de Janeiro, Brasil.

2) Pesquisador do CNPq. E-mail: gerson@ufrrj.br

3) Curso de Graduação em Ciências Biológicas. Bolsista Iniciação Científica CNPq.

E-mail: rnvicentini@bol.com.br 
freqüência de comprimento, e como medida da variação do peso esperado para o comprimento de um indivíduo, indicando sua condição, ou seja, o acúmulo de gordura e desenvolvimento gonadal (Rossi-WoNGTSCHOWSKI 1977). O objetivo do presente trabalho foi determinar a relação peso-comprimento para a população de corvinas da Baía de Sepetiba, visando contribuir com o conhecimento da sua biologia, em seus aspectos de crescimento relativo, bem como estabelecer comparações com outras populações de corvina da costa brasileira.

\section{MATERIAL E MÉTODOS}

\section{Área de estudo}

A Baía de Sepetiba localiza-se ao sul do Estado do Rio de Janeiro, e apresenta área de $305 \mathrm{~km}^{2}$, drenando uma bacia de $3500 \mathrm{~km}^{2}$ (Fig. 1). Apesar de atingir até 30 metros de profundidade nas proximidades do limite com o mar, apresenta profundidades inferiores a cinco metros em $40 \%$ de sua área, com substrato predominantemente lodoso. É caracterizada por apresentar salinidade em torno de $30 \%$ ao longo de todo o ano, e temperatura média variando entre $21^{\circ} \mathrm{C}$ no inverno e $28^{\circ} \mathrm{C}$ no verão (BARBIÉRE \& KRONEMBERGER 1994).

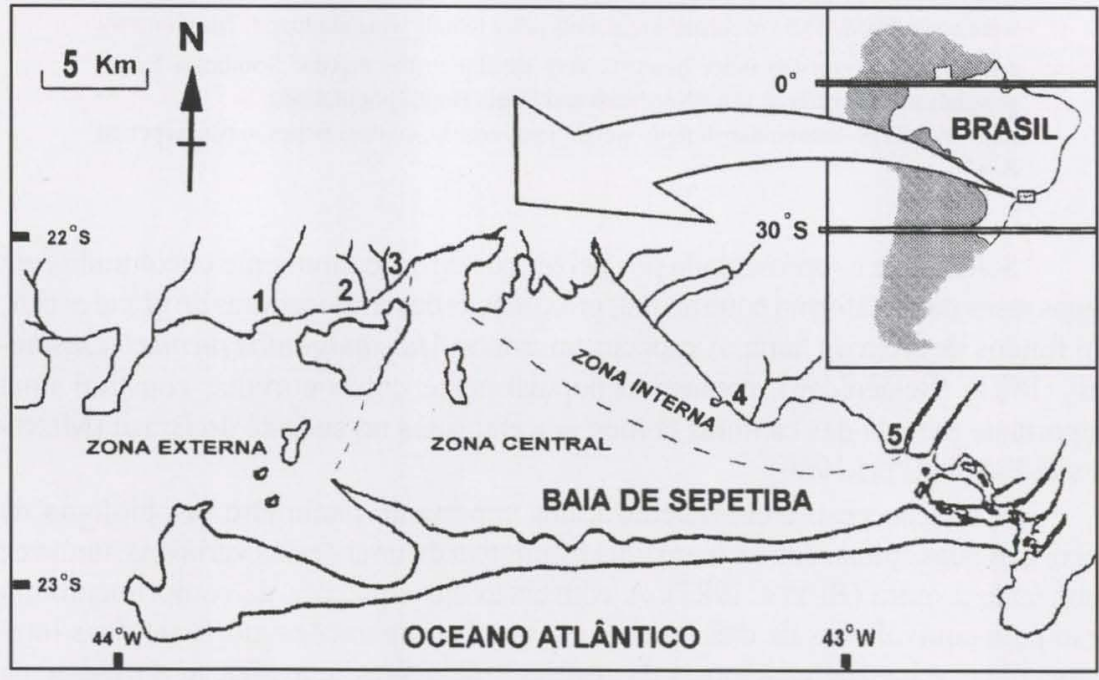

Fig. 1. Mapa da área de estudo, Baía de Sepetiba, Rio de Janeiro. Locais de coleta de arrasto de praia: (1) Muriqui; (2) Itacuruçá; (3) Coroa Grande; (4) Sepetiba; (5) Guaratiba. Arrasto de fundo: zonas interna, central e externa.

Climaticamente, a Baía enquadra-se no grupo A da classificação, definido como clima tropical, diferenciando-se em função do contraste de topografia entre a área plana da baixada e as encostas que a envolvem. As chuvas concentram-se em dezembro-março e o período mais seco, de maio a setembro (BARBIÉRE \& KRONEMBERGER 1994). Ventos sudoeste e nordeste alternam-se como predominantes 
na área. A entrada de águas oceânicas frias e densas, provenientes da Corrente das Malvinas, dá-se à oeste da Baia; ao misturarem-se com as águas doces dos rios na zona interna, as correntes aquecem-se e tornam-se superficiais, contornando a Baia e saindo pelo canal principal, onde existem sobreposições de correntes superficiais que saem e profundas que entram (COELHO \& CARVALHO 1973).

\section{Analise de dados}

Foram utilizados 971 exemplares de M. furnieri, provenientes de dois programas de amostragens; um de arrasto de fundo e outro de arrasto de praia, realizados na Baía de Sepetiba, Rio de Janeiro, no período de outubro de 1998 a maio de 1999 (arrasto fundo) e, de outubro a novembro de 1998 (arrasto praia). Os arrastos de fundo foram realizados com barco do tipo arrasteiro de $12 \mathrm{~m}$ de comprimento com três amostragens aleatórias em cada zona - interna, central e externa - da Baía. Os arrastos de praia foram realizados em cinco locais de coleta ao longo da margem continental da Baía de Sepetiba (Fig. 1). Os peixes foram identificados com o auxílio do trabalho de MENEZES \& Figueiredo (1980), medidos no comprimento total (em milímetros), pesados (em gramas) e fixados em formol a $10 \%$, sem a determinação do sexo. Para a relação peso-comprimento foi aplicada a fórmula: $\mathrm{Wt}=\mathrm{a} \mathrm{Lt}^{\mathrm{b}}$ (LE CREN 1951), onde Wt corresponde ao peso, Lt, ao comprimento, a, ao fator relacionado com o grau de engorda dos indivíduos e b $(=\theta)$, ao coeficiente de alometria, relacionado com a forma do crescimento dos indivíduos. Os valores de a e b foram estimados a partir do método dos mínimos quadrados (modelo preditivo), após transformação logarítmica na base neperiana da seguinte equação: $\ln \mathrm{Wt}=\ln \mathrm{a}+\mathrm{b} \ln \mathrm{L} \mathrm{t}$. O fator de condição $(\varnothing)$ foi calculado através da expressão $\varnothing=\mathrm{Wt} / \mathrm{Lt}^{\ominus}$ (SANTOS 1978).

\section{RESULTADOS E DISCUSSÃO}

Foram utilizados peixes compreendidos entre a faixa de 15 a $345 \mathrm{~mm}$ de comprimento total, e peso total de 0,04 a $600 \mathrm{~g}$. A relação peso-comprimento determinada foi a seguinte: $\mathrm{Wt}=0,00000942 \mathrm{Lt}^{2,99}$ (Fig. 2). Essa equação corresponde à forma logaritimizada, $\ln \mathrm{Wt}=-11,57+2,996 \ln \mathrm{Lt}(\mathrm{r}=0,99)$.

$O$ fator de condição $\varnothing$, relacionado ao bem-estar ou índice de gordura do peixe, geralmente é maior quando associado a indivíduos que apresentam maiores pesos para um dado comprimento. Já o coeficiente de alometria $\theta$ indica a velocidade de inflexão da curva para atingir os valores assintóticos, isto é, quando o crescimento passa a apresentar um incremento irrelevante em relação ao peso (LE CREN 1951). Em alguns casos, o valor de $\varnothing$ diminui quando a freqüência de indivíduos maduros é elevada (VAZZOLER \& VAZZOLER 1965) ou quando a gônada está em seu máximo de maturidade (CONAND 1977), representando, nestes casos, mais um indicador de períodos de alimentação e jejum, que podem também estar ligados ao ciclo reprodutivo. Em outros casos, não se verifica qualquer relação entre a condição e o ciclo gonadal (BLACKBURN 1960). O fator de condição, em função de sua variação, constitui-se em elemento quantitativo importante para a avaliação da relação entre 
a forma do corpo e seu peso relativo. O desenvolvimento das gônadas femininas ocorre em ritmo mais intenso que o incremento, em comprimento ou peso do peixe, tendo, como consequiência, uma mudança mais acentuada na forma do corpo das fêmeas ao longo do ciclo sexual. Isso se manifesta com um aumento do peso total e, consequentemente, do $\varnothing$ de caráter temporário, quando a gônada atinge seu máximo tamanho, antes da desova (ANGELESCU et al. 1958).

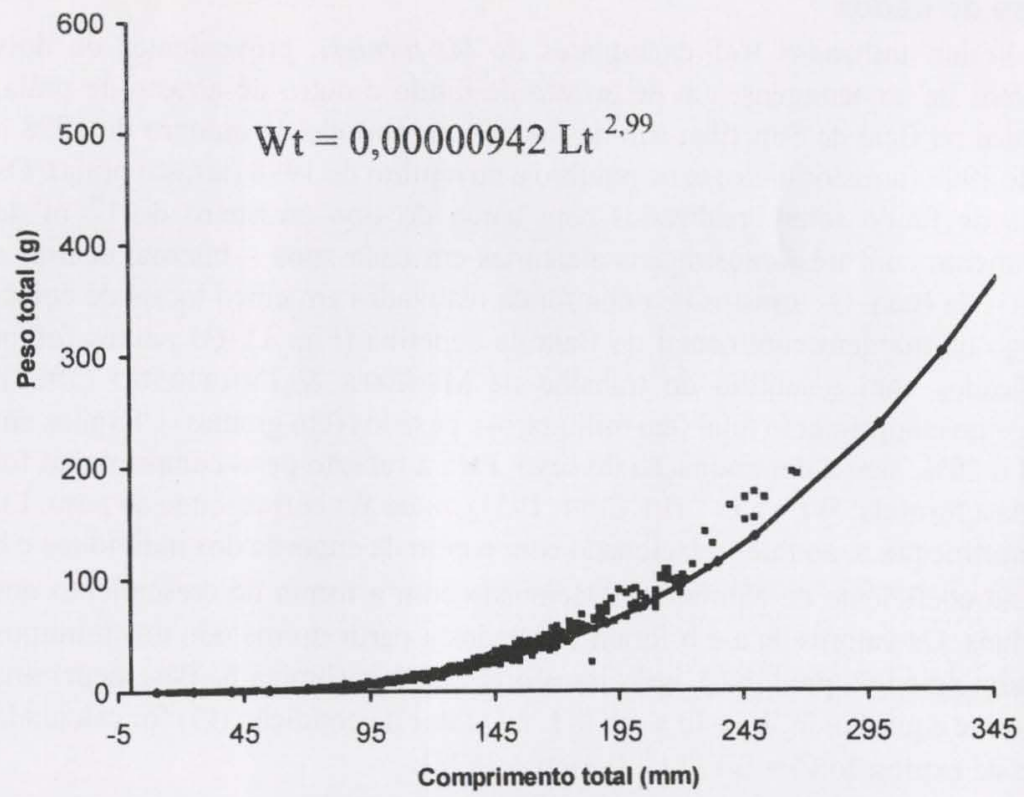

Fig. 2. Relação peso-comprimento de Micropogonias furnieri na Baia de Sepetiba, Rio de Janeiro.

Comparando-se o fator de condição $\varnothing\left(0,942 \times 10^{-5}\right)$ do presente trabalho com o encontrado por ISAAC-NAHUM \& VAZZOLER (1983) na população de corvina de Ubatuba, São Paulo $\left(\varnothing=0,8511 \times 10^{-5}\right)$ e com as populações da zona costeira ao sul de Cabo Frio, Rio de Janeiro (VAZZOLER 1971) onde $\varnothing=1,03 \times 10^{-5}$, verificou-se que a população da Baía de Sepetiba apresentou valores próximos aos destas populações. Em relação às populações da Lagoa dos Patos-RS (CASTELlo 1986), onde $\varnothing=0,51 \times 10^{-5}$, as corvinas da Baía de Sepetiba apresentaram números mais elevados. Essa diferença talvez ocorra pelo fato do ambiente estuarino da Lagoa dos Patos ser mais estressante osmoticamente, comparado às condições ambientais mais estáveis da Baía de Sepetiba e das zonas costeiras, e com isso impulsionarem uma demanda de parte da energia dos peixes que seria utilizada no seu crescimento.

Em geral os valores de $\theta$ se aproximam de 3, o que foi verificado no presente estudo, caracterizando o crescimento alométrico desta espécie. LE CREN (1951) afirma que os valores de $\theta$ variam de 2,0 a 4,0, assumindo o valor 3,0 para um "peixe ideal", que mantém a mesma forma durante o crescimento ontogenético. Valores 
inferiores ou superiores a 3,0 indicam indivíduos que, ao longo do crescimento, se tornam mais "longilineos" ou "redondos", respectivamente.

$O$ coeficiente de alometria $\theta$ apresentou valores aproximados ao da população do sul de Cabo Frio $(\theta=2,99)$ determinado por VAzzolER (1971) e ao da população de Ubatuba $(\theta=3,03)$, porém se apresentou menor do que o coeficiente encontrado por JURAS (1984 apud VAZZOLER 1991) na costa maranhense $(\theta=3,05$ fêmeas e 3,09 machos) e por CASTELlo (1986) na Lagoa dos Patos, Rio Grande do $\operatorname{Sul}(\theta=3,15)$. A ocorrência destas últimas duas populações, associadas a ambientes estuarinos, onde existe maior disponibilidade de alimento comparada com as populações de ambientes costeiros poderia estar determinando os maiores valores deste coeficiente de alometria (VAZZOLER 1991).

AGRADECIMENTOS. Este trabalho foi realizado com apoio do Conselho Nacional de Desenvolvimento Científico e Tecnológico, CNPq, através de bolsas e auxílio-pesquisa ao Projeto "Bioecologia dos peixes da Baia de Sepetiba".

\section{REFERÊNCIAS BIBLIOGRÁFICAS}

ANGELESCU, V.; F.S. GNERI \& A. NANI. 1958. La merluza del mar argentino (biologia y taxonomia) Serv. Hidrog. Nav., Buenos Aires, (H 1004): 1-224.

Barbiére, E.B. \& D.M.P. Kronemberger. 1994. Climatologia do Litoral Sul-Sudeste do Estado do Rio de Janeiro. Cad. Geoc., Rio de Janeiro, 12: 57-73.

BEYER, J.E. 1987. On lenght-weight relationships. Part 1: computing the mean weight of the fish of a given lenght class. Fishbyte 5: 11-13.

BLACKBURN, M. 1960. A study of condition (weight for length) of Australian barracouta Thyrsites atum (Eupharasen). Austr. Jour. Mar. Freshw. Res. 11 (1): 14-41.

Bolger, T. \& P.L. Connolly. 1989. The selection of suitable indices for the measurement and analysis of fish condition. Jour. Fish Biol. 34: 171-182.

Castello, J.P. 1986. Distribuición, crescimiento y maturación sexual de la corvina juvenil (Micropogonias furnieri) en el estuário de la "Lagoa dos Patos", Brasil. Physis 44 (106): 21-36.

Coelho, V.M.B. \& R.R. Carvalho. 1973. Levantamento Sanitário da Baía de Sepetiba e suas Possibilidades como Corpo Receptor de Cargas Poluidoras da Região. Rio de Janeiro, Publicações Avulsas FEEMA, 87p.

Conand, C. 1977. Contribution à l'etude du cycle sexual et de la fecundité de la sardinella ronde, Sardinella aurita: pêche sardiniere da karoise en 1975, et premier semestre 1976. Cah. O.R.S.T.O.M., Sér. Océanogr. 15 (14): 301-312.

ISAAC-NAHUM, V.J. \& A.E.A. DE M. VAZZOLER. 1983. Biologia reprodutiva de Micropogonias furnieri (Desmarest, 1828) (Teleostei, Sciaenidae). 1. Fator de condição como indicador do período de desova. Bol. Inst. Oceanogr., São Paulo, 32 (1): 63-69.

LE-CREN, E.D. 1951. The lenght-weight relationship and seasonal cycle in gonad weight and conditions in the perch Perca fluviatilis. Jour. Anim. Ecol. 20 (2): 201-1.

Menezes, N.A. \& J.L. Figueiredo. 1980. Manual de peixes marinhos do sudeste do Brasil. IV Teleostei (3). São Paulo, Museu de Zoologia, Univ. São Paulo, 96p.

Rossi-WoNGTSCHOWSKI, C.L.B. 1977. Estudo das variações da relação peso total/ comprimento total em função do ciclo reprodutivo e comportamento de Sardinella brasiliensis (Steindachner,1879) da costa do Brasil entre $23^{\circ} \mathrm{S}$ e $28^{\circ} \mathrm{S}$. Bol. Inst. Oceanogr. 26: 131-180. 
SANTOS, E.P. DOS. 1978. Dinâmica de populações aplicada à pesca e psicultura. São Paulo, Hucitec, Ed. Univ. São Paulo, 129p.

VAZZOLER, A.E.A. DE M. 1971. Diversificação fisiológica e morfológica de Micropogonias furnieri (Desmarest, 1822) ao Sul de Cabo Frio, Brasil. Bol. Inst. Oceanogr. 20 (2): 1-70. 1991. Síntese de conhecimentos sobre a biologia da corvina, Micropogonias furnieri (Desmarest, 1823), da costa do Brasil. Atlântica, Rio Grande, 13 (1): 55-74.

VAzzoler, A.E.A. DE M. \& G. Vazzoler. 1965. Relation between condition factor and sexual development in Sardinella aurita (Cuv. \& Val.). Anais Acad. Bras. Ciênc. 37 (Supl.): 353-359.

Recebido em 30.III.2000; aceito em 29.III.2001. 